\title{
Bound-Electron Nonlinearity Beyond the Ionization Threshold
}

\author{
J. K. Wahlstrand, ${ }^{1,2}$ S. Zahedpour, ${ }^{1}$ A. Bahl, ${ }^{3}$ M. Kolesik, ${ }^{3}$ and H. M. Milchberg ${ }^{1, *}$ \\ ${ }^{1}$ Institute for Research in Electronics and Applied Physics, University of Maryland, College Park, Maryland 20742, USA \\ ${ }^{2}$ Engineering Physics Division, National Institute of Standards and Technology, Gaithersburg, Maryland 20899, USA \\ ${ }^{3}$ College of Optical Sciences, University of Arizona, Tucson, Arizona 85712, USA
}

(Received 10 February 2018; published 2 May 2018)

\begin{abstract}
We present absolute space- and time-resolved measurements of the ultrafast laser-driven nonlinear polarizability in argon, krypton, xenon, nitrogen, and oxygen up to ionization fractions of a few percent. These measurements enable determination of the strongly nonperturbative bound-electron nonlinear polarizability well beyond the ionization threshold, where it is found to remain approximately quadratic in the laser field, a result normally expected at much lower intensities where perturbation theory applies.
\end{abstract}

DOI: $10.1103 /$ PhysRevLett.120.183901

The nonlinear dipole moment per unit volume $\left(\mathbf{P}_{\mathrm{NL}}\right)$, driven by strong laser fields beyond the perturbative limit in atoms and molecules, can play a central role in the propagation of intense ultrashort optical pulses in material media. It is integral to the process of high harmonic generation and its phase matching [1,2]. It leads to extremely wide bandwidths in supercontinuum generation [3], which can be applied to single-cycle pulse generation [4] and ultrafast spectral interferometry [5]. The space and time dependence of $\mathbf{P}_{\mathrm{NL}}$ also controls the collapse and collapse arrest of optical beams in femtosecond filamentation [6,7]. In this regime, the optical field is no longer a small perturbation on the atomic potential; it is of comparable strength.

While extensive absolute measurements of $\mathbf{P}_{\mathrm{NL}}$ have been done in neutral atomic and molecular gases below the ionization threshold [8-10], the nonlinear response above the ionization threshold is composed of contributions from the free electrons and the residual neutrals, where the free electron response strongly masks that of the bound electrons. Separating out these contributions requires determination of the absolute transient ionization rate. It is such measurements that will complete the picture of the nonlinear polarization in intense nonperturbative fields.

In this Letter, we fully map the nonlinear polarization of several atomic and molecular gas species through the ionization transition with sufficient accuracy to reveal the bound contribution surviving above the ionization threshold. For the surviving atoms (molecules), the nearinstantaneous bound nonlinear electronic response to a laser field $\mathbf{E}$ can be written $\mathbf{P}_{\mathrm{NL}}^{b}=\Delta \chi_{b} \mathbf{E}=\left(\Delta n_{b} / 2 \pi\right) \mathbf{E}$, where $\Delta \chi_{b}=N \alpha_{\mathrm{NL}}$ is the bound-electron nonlinear susceptibility, $N$ is the gas density, $\alpha_{\mathrm{NL}}$ is the nonlinear polarizability, and $\Delta n_{b}=2 \pi N \alpha_{\mathrm{NL}}$ is the laser fieldinduced nonlinear refractive index shift. We find that, in all gases studied, the relationship between the bound electronic response and laser field observed at lower intensities [8,9], $\Delta n_{b} \propto|\mathbf{E}|^{2}$, extends to intensities where up to $\sim 10 \%$ of atoms or molecules are ionized, a region significantly beyond the limits of perturbation theory [11].

Prior measurements of the ionization yields of atoms and molecules by intense ultrashort optical pulses were performed in vacuum chambers at very low pressure. In those experiments, ionization by-products (electrons and ions) are directly captured long after the ionizing pulse has passed through the interaction volume, approximately the beam waist region $[12,13]$, and ionization yields are scaled and compared to space and time integrations of ionization rate models. Those experiments were not intended to address the absolute nonlinear polarizability of atoms and molecules in intense fields.

There has recently been considerable discussion about the effect of strongly nonperturbative fields on the atomic or molecular dipole response underlying nonlinear propagation. In particular, debate has arisen (see, for example, [14] and references therein) regarding potentially exotic contributions $[14,15]$ to the dipole moment, wherein strongly driven bound electrons have been speculated to exhibit a negative polarizability, with this scenario advanced to explain collapse arrest in femtosecond filamentation [16-18]. This debate has persisted due to both experimental and theoretical complications. Experimentally, at high fields, propagation effects of ionization have been very difficult to avoid. Theoretically, it has been challenging to cleanly separate the contributions of bound and free electrons $[15,19,20]$. Here, we present new experimental results showing beyond any doubt that the nonlinear bound response continues to increase approximately quadratically with the laser field (or linearly with the intensity), even in the presence of substantial and increasing ionization.

A diagram of the experiment is shown in Fig. 1. The experiment employs single-shot supercontinuum spectral interferometry (SSSI) $[5,21]$, which measures, in a single shot, the transient refractive index shift $\Delta n$ induced by an 


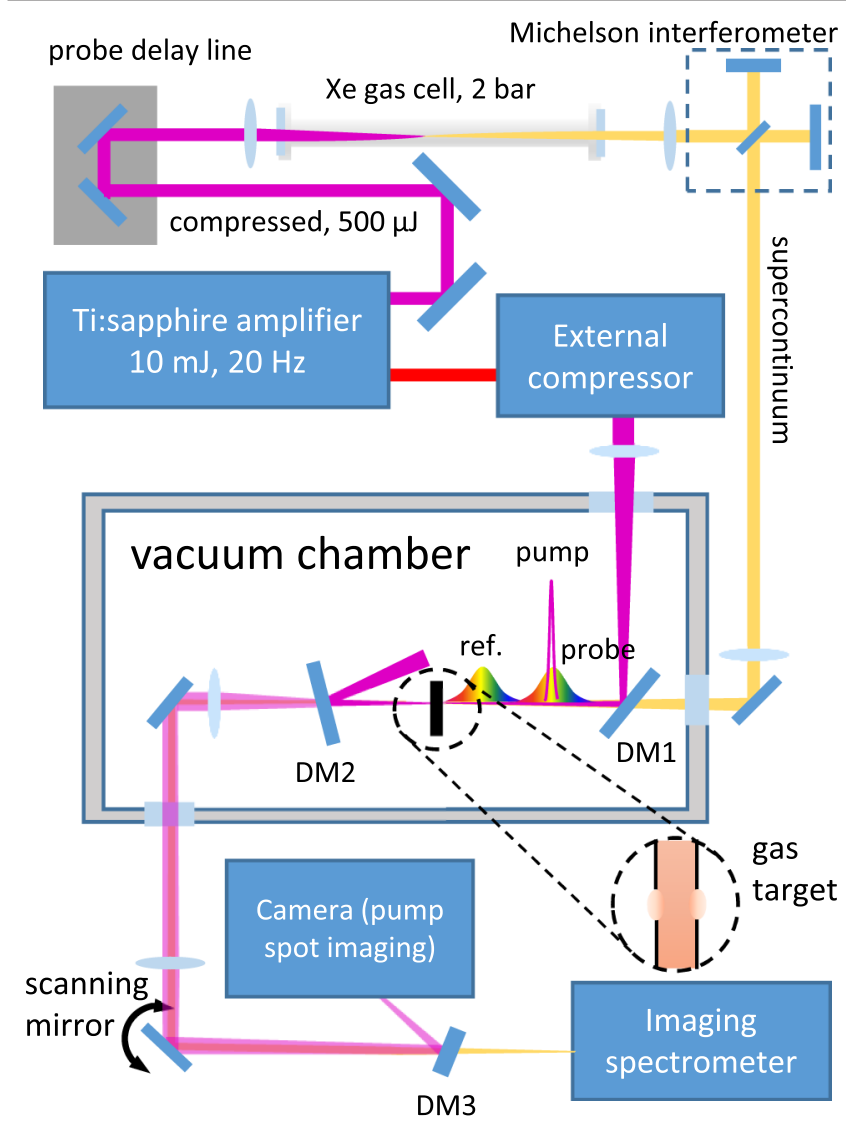

FIG. 1. Experimental apparatus for 2D + 1 SSSI measurement of field-induced ionization. DM1, DM2, DM3: dichroic mirrors. Not shown: $800 \mathrm{~nm}$ rejection mirror after Xe gas cell, auxiliary interferometer for gas target characterization, and pretarget pump spot imaging camera.

intense pump pulse in a medium. Broadband supercontinuum (SC) probe and reference pulses $(\Delta \lambda>100 \mathrm{~nm})$ are copropagated with the pump pulse into a very thin gas target, with the pump and probe temporally overlapped and the reference preceding them. The probe picks up a phase shift $\Delta \Phi=k \Delta n L_{\text {eff }}$ from the pump-induced refractive index change $\Delta n$ in the gas, where $k$ is the vacuum wave number. The effective gas target thickness $L_{\text {eff }}=$ $\int\left[N(z) / N_{0}\right] d z \sim 450 \mu \mathrm{m}$ satisfies $L_{\text {eff }} / z_{p \text {,pr }} \ll 1$, where $N(z)$ is the gas density profile along the optical path, $N_{0}$ is the profile mean density, and $z_{p \text {,pr }}$ is the pump (probe) Rayleigh length $[8,22]$. The probe and reference pulses are imaged from the interaction region to the entrance slit of an imaging spectrometer, forming a spectral interferogram. Analysis of the interferogram yields the probe phase and amplitude shifts resolved in time and one transverse spatial dimension (along the slit $x$ ), yielding $\Delta n=\Delta n(x, t)$. The technique provides sub-10 fs time resolution set by the inverse probe bandwidth and few micron scale spatial resolution $[5,21]$.

A new scheme, which we call $2 \mathrm{D}+1$ SSSI, yields $\Delta n(x, y, t)$. Here, the image of the SC beam in the thin gas

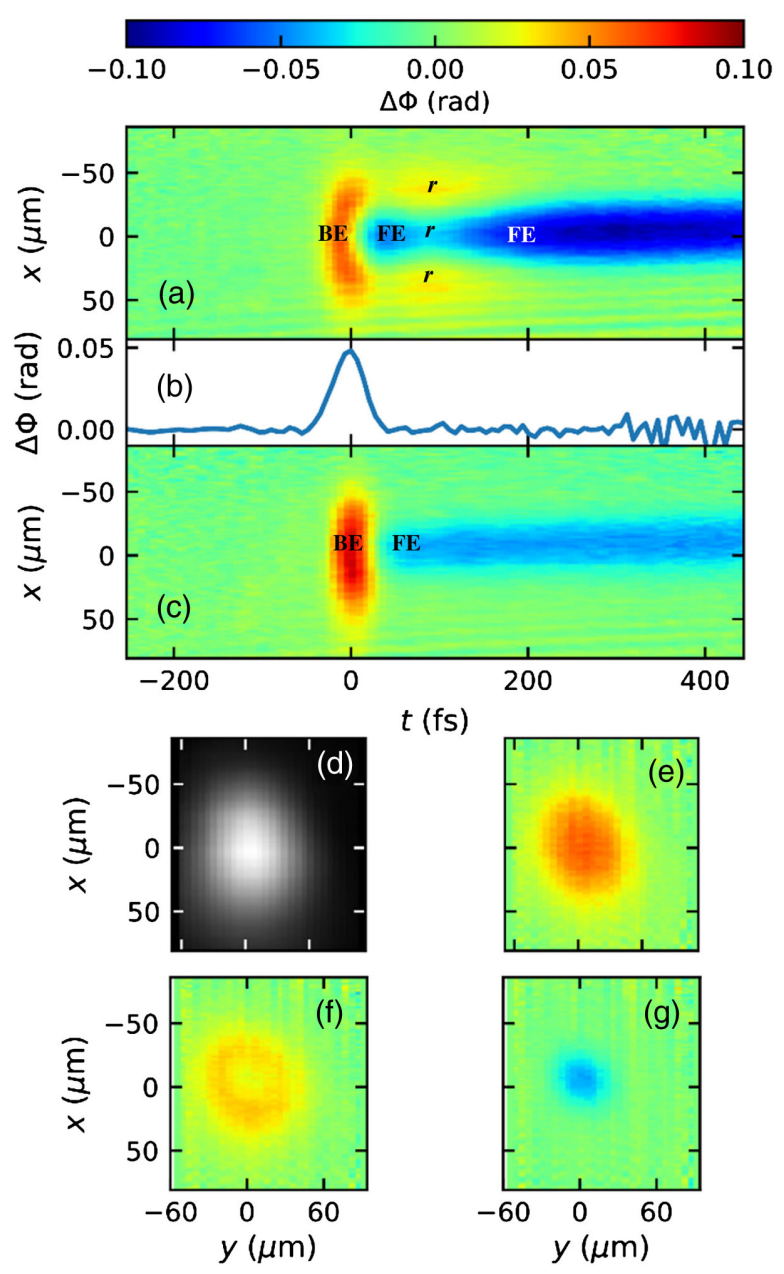

FIG. 2. Results in Ar for peak pump intensity of $95 \mathrm{TW} / \mathrm{cm}^{2}$ and in $\mathrm{N}_{2}$ for peak pump intensity of $115 \mathrm{TW} / \mathrm{cm}^{2}$. The pulse width is 42 fs. (a) $1 \mathrm{D}+1$ phase shift $\Delta \Phi\left(x, y_{0}=0, t\right)$ in $\mathrm{N}_{2}$. The complex structure results from the bound-electronic (BE), rotational $(r)$, and free electron (FE) contributions to the transient polarizability. (b) Pump pulse envelope given by the Kerr phase shift $\Delta \Phi\left(x_{0}=0, y_{0}=0, t\right)$ in $\mathrm{Ar}$ at $47 \mathrm{TW} / \mathrm{cm}^{2}$. (c) $1 \mathrm{D}+1$ phase shift $\Delta \Phi\left(x, y_{0}=0, t\right)$ in Ar, showing BE and FE contributions. (d) Image of the pump spot at the gas target for Ar data. 2D +1 SSSI-derived phase shift images in Ar: (e) $\Delta \Phi(x, y, t=-14 \mathrm{fs})$, dominated by the Kerr response, (f) $\Delta \Phi(x, y, t=+25 \mathrm{fs})$, showing the Kerr response on the wings and the growing plasma contribution in the center of the beam; and (g) $\Delta \Phi(x, y, t=+100 \mathrm{fs})$, showing the dominant plasma contribution after the pump pulse. A movie of $\Delta \Phi(x, y, t)$ is provided in [27]. The peak of the pump pulse defines zero for the $x, y$, and $t$ coordinates.

target is scanned perpendicular to the spectrometer slitalong $y$-by a motorized scanning mirror downstream of the second imaging lens. Each captured shot yields a spectral interferogram at a particular value of $y\left(=y_{i}\right.$, say). Multiple interferograms are averaged before extraction to significantly improve the signal-to-noise ratio [23,24], yielding $\Delta \phi\left(x, y_{i}, \omega\right)$ and then $\Delta \Phi\left(x, y_{i}, t\right)$ [21]. A $2 \mathrm{D}+1$ map 
$\Delta \Phi(x, y, t)=k \Delta n(x, y, t) L_{\text {eff }}$ is built over $\sim 10^{4}$ shots by scanning $y$. An important feature of $2 \mathrm{D}+1$ SSSI is that a single 2D phase profile at a particular time slice encodes the response over a wide range of intensity, greatly improving the statistics of our measurements.

Figures 2(a) and 2(c) show 1D +1 SSSI traces for ionization of $\mathrm{N}_{2}$ and Ar. The more complex $\mathrm{N}_{2}$ trace shows the prompt electronic (Kerr) response, a delayed positive alignment rotational response (which is larger than the Kerr contribution [8]), followed by molecular antialignment (alignment) and plasma contributions. The Ar trace shows the early Kerr response followed by the rapid tunnel ionization to long-lived plasma. Figure 2(b) is a lineout of the Kerr response in Ar below the onset of ionization, which gives the pump intensity envelope. Temporal slices of $2 \mathrm{D}+1 \Delta \Phi(x, y, t)$ traces in Ar are shown in Figs. 2(e)-2(g) for a 42 fs pump pulse of peak intensity $95 \mathrm{TW} / \mathrm{cm}^{2}$ and show the whole beam spatial effect of the positive and negative transient index contributions. Unlike in our previous results [23-25], these new measurements are absolute and quantitative at intensities where ionization is observed. This required increasing the pump spot size to minimize refraction of the pump and probe by the plasma transverse gradient, increasing the time between pump pulses to $100 \mathrm{~ms}$ to avoid cumulative thermal effects in the gas [26], improving the temporal resolution of SSSI by minimizing the probe chirp (consistent with the desired temporal window), and optimizing the spectrometer resolution [21]. Results for the other gases studied, including movies of $\Delta \Phi(x, y, t)$, are provided in the Supplemental Material [27].

We first examine the pure plasma component of the phase shift, which can be isolated by examining time delays long after contributions by the bound electron response, which includes the prompt Kerr response and, in the case of $\mathrm{N}_{2}$ and $\mathrm{O}_{2}$, the delayed rotational response [24,25]. For our case of a thin gas target in which the probe experiences negligible refraction, the refractive index profile $\Delta n(x, y, t)=\Delta \Phi(x, y, t) / k L_{\text {eff }}$ gives $N_{e}(x, y)=-2 N_{\mathrm{cr}} \Delta n\left(x, y, t_{1}\right)$ for the axially averaged electron density profile, where $t_{1}>50 \mathrm{fs}$ for the noble gases and $t_{1}>250$ fs for $\mathrm{N}_{2}$ and $\mathrm{O}_{2}$. Here we have used the refractive index shift induced by a low density collisionless plasma $\Delta n=-N_{e} / 2 N_{\mathrm{cr}}$, where $N_{\mathrm{cr}}=3.1 \times$ $10^{21} \mathrm{~cm}^{-3}$ is the critical electron density at the probe central wavelength $\lambda_{\mathrm{pr}} \approx 600 \mathrm{~nm}$. For negligible probe refraction, each probe ray centered at $\left(x_{i}, y_{i}\right)$ samples the dynamics induced by the intensity profile $I\left(x_{i}, y_{i}, t\right)$. The ionization yield $Y=N_{e} / N_{0}$ as a function of intensity is shown in Fig. 3. For each data set (a complete $2 \mathrm{D}+1$ scan of $\sim 10^{4}$ consecutive shots at the same nominal peak laser power), $Y$ (for times $t>t_{1}$ ) was sorted into 20 intensity bins. The points in Fig. 3 are average values for $Y$ in each bin.
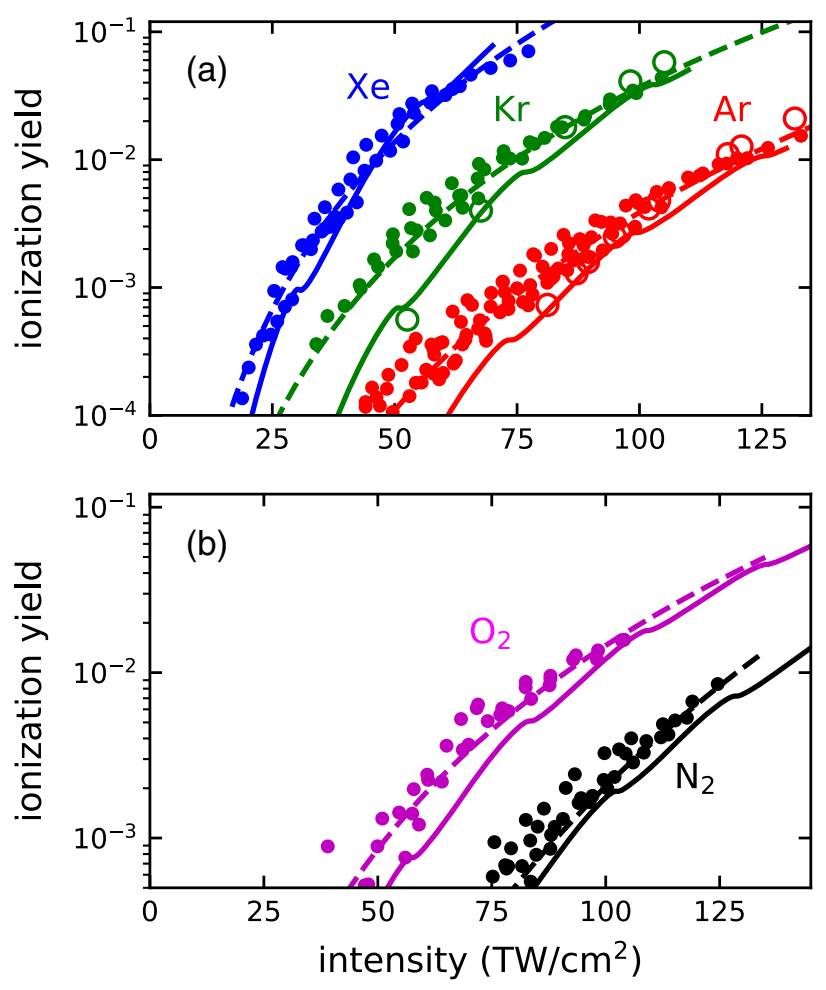

FIG. 3. Ionization yield (points) as a function of peak intensity $I_{0}$ for (a) $\mathrm{Ar}$ (red), $\mathrm{Kr}$ (green), $\mathrm{Xe}$ (blue) and (b) $\mathrm{N}_{2}$ (black) and $\mathrm{O}_{2}$ (magenta). PPT [28] rates are shown as solid lines. MESA calculations for $\mathrm{Ar}$ and $\mathrm{Kr}$ are shown as large open circles [29,30]. Fits to $Y=c_{1} I_{0}^{m}$, described in [27], are shown as dashed lines, where $c_{1}$ and $m$ are determined from the fit. The accuracy of the ionization yield measurement is set by the vertical scatter of the points, while the intensity accuracy is $\sim 13 \%$ as discussed in the main text.

As in most measurements of the nonlinear response, the error is dominated by the uncertainty in the peak intensity. Here, in a new procedure, we use our previous measurement of the nonlinear refractive index of $\mathrm{Ar}$, $n_{2}=(9.7 \pm 1.2) \times 10^{-20} \mathrm{~cm}^{2} / \mathrm{W}$, applicable for intensities to nearly the ionization threshold [9], to provide full 2D calibration of our intensity profiles through $\Delta n(x, y)=$ $2 n_{2} I(x, y)$ by directly comparing CCD camera images of the pump spot to 2D Kerr phase shift profiles measured with $2 \mathrm{D}+1$ SSSI at subionization intensities of $<50 \mathrm{TW} / \mathrm{cm}^{2}$. Intensities above this level, for which the focal spot and pulse width remain the same, are determined through scaling by the pulse energy. The uncertainty in the intensity is $13 \%$, mostly arising from the uncertainty in $n_{2}$, with the residual uncertainty due to shot-to-shot fluctuations in the measured phase shift of $\sim 3$ mrad.

We compare our results to two ionization models. The single-active-electron Perelomov-Popov-Terent'ev (PPT) model [28] (solid lines) shows ionization yields in reasonable agreement with the curves of Fig. 3. We also performed a full simulation of the pump-probe experiment using the unidirectional pulse propagation equation $[29,30]$ 

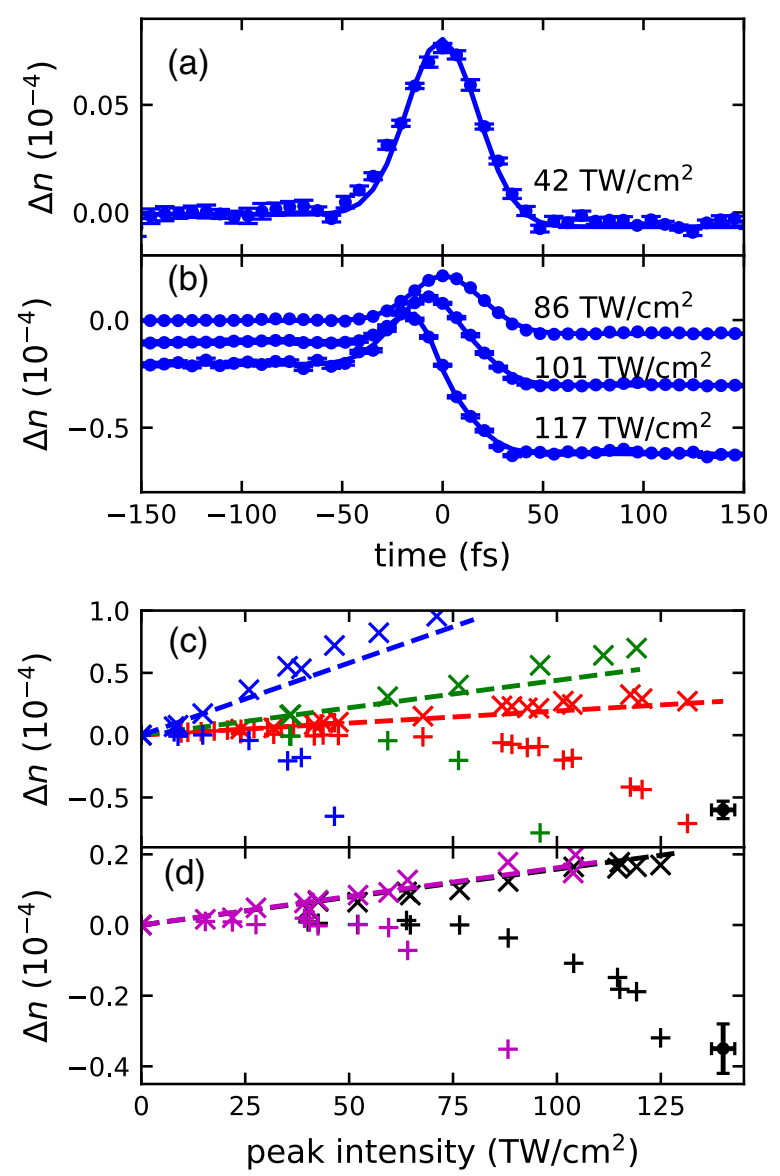

FIG. 4. Response during pump pulse. (a) Nonlinear index shift in Ar vs time for peak intensity $42 \mathrm{TW} / \mathrm{cm}^{2}$ (dots), below the threshold for ionization, fit to a Gaussian pulse with $\tau_{\mathrm{FWHM}}=$ $42 \mathrm{fs}$ (solid line). (b) Nonlinear index shift vs time and fits to the standard model (Kerr effect plus ionization) for Ar (solid lines). The curves have been offset vertically for clarity. (c) Plot of Kerr index change $\Delta n_{K}(\times)$ and plasma index change $\Delta n_{p}(+)$ from fits as a function of intensity in $\mathrm{Ar}$ (red), $\mathrm{Kr}$ (green), and $\mathrm{Xe}$ (blue). (d) Same as (c) for $\mathrm{N}_{2}$ (black) and $\mathrm{O}_{2}$ (magenta). The black points on the lower right of (c) and (d) indicate typical uncertainty. Dashed lines show the extrapolated Kerr response $2 n_{2} I$ from previous low intensity measurements [8,9].

to model pump and probe propagation and the metastable electronic state approach (MESA) [31-33] to model the full nonlinear response. The results, shown as open circles, are in similarly reasonable agreement with the experimental curves for $\mathrm{Ar}$ and $\mathrm{Kr}$ [31]. Detailed comparisons of the measured spatiotemporal nonlinear response and MESA simulations, which largely agree, are described in a separate publication [33].

The measured time-dependent index shift in argon $\Delta n(x=0, y=0, t)=\Delta n(t)$, from the onset of the Kerr response through ionization, is shown in Figs. 4(a) and 4(b). Similar figures for the other gases are found in [27]. At low intensity, the response follows the pump pulse intensity envelope, which is well fit by $I(t)=I_{0} e^{-t^{2} / \tau^{2}}$, where $\tau=\tau_{\mathrm{FWHM}} /(2 \sqrt{\ln 2})$ corresponds to our pulse full width at half maximum $\tau_{\mathrm{FWHM}}=42$ fs [Fig. 4(a)]. Figure 4(b) shows the time-dependent refractive index shift in $\mathrm{Ar}$ for increasing intensity and ionization levels, along with fits to $\Delta n(t)=\Delta n_{K} e^{-t^{2} / \tau^{2}}+\Delta n_{p}(1+$ $\left.\operatorname{erf}\left[m^{1 / 2} t / \tau\right]\right) / 2$, where $\Delta n_{K}$ is a fitting parameter and $\Delta n_{p}$ is the peak plasma index shift. The first term, modeling the bound response $\Delta n_{b}(t)$, is a Kerr-like (instantaneous) response for a Gaussian pulse of peak intensity $I_{0}$, where $\Delta n_{K}=2 n_{2} I_{0}$ is the peak index shift experienced by a probe pulse [8]. The second term models the plasma contribution as $N_{e}(t) / N_{0}=Y(t) \approx \int_{-\infty}^{t} w\left(t^{\prime}\right) d t^{\prime}$ using an ionization rate $w(t)=c_{2}\left(I_{0} e^{-t^{2} / \tau^{2}}\right)^{m}$, for which the yield is $Y(t \rightarrow \infty)=c_{1} I_{0}^{m}$, and where $c_{1}$ and $m$ are determined from fits to the ionization curves in Fig. 3 and $\Delta n_{p}=$ $-N_{0} c_{1} I_{0}^{m}\left(2 N_{\text {cr }}\right)^{-1}$ [27]. The approximate reduction in Kerr response due to the reduction of the neutral atom density by ionization is accounted for by multiplying the $\Delta n_{K}$ value found from the fit by $1-Y / 2$. This adjustment, which assumes that the Kerr response from the ions is negligible, reduces $\Delta n_{K}$ by at most $3 \%$ at the highest intensity. This simple model is seen to be an excellent fit to the measured transient index shift. The point of the expression used for $Y$ is not to advance a multiphoton-ionization (MPI)-like model for ionization; it is to provide an analytic model fit to the ionization yield data to enable separation of the bound and free electron contributions. In fact, as seen in [27], the best fit values for $m$ are notably smaller than their corresponding MPI values for each species, indicating the significant contribution of tunneling ionization.

Figures 4(c) and 4(d) plot, as a function of peak intensity, the peak Kerr index shift $\Delta n_{K}$ and the peak plasma shift $\Delta n_{p}$ extracted from fits to transient index data, as in Fig. 4(b), for each species, with Fig. 4(c) showing atomic results and Fig. 4(d) showing molecular results. Remarkably, it is seen for all species that $\Delta n_{K}$ continues to rise with intensity even as $\Delta n_{p}$ becomes increasingly negative up to the limit of our measurements. In $\mathrm{Ar}$, $\mathrm{Kr}$, and $\mathrm{Xe}$, the Kerr index shift observed above the ionization threshold is somewhat higher than the curve extrapolated from below the threshold [9], shown as a dashed line in Figs. 4(c) and 4(d). Apparently, $\Delta n_{K}$ rises slightly faster than $\propto|\mathbf{E}|^{2}$ in the noble gases, but confirmation awaits more accurate measurements. In the molecular gases, the result is somewhat different. In $\mathrm{N}_{2}$, the Kerr index shift above the ionization threshold closely follows the $\propto|\mathbf{E}|^{2}$ curve extrapolated from the subthreshold response [8], while in $\mathrm{O}_{2}$ it is below the extrapolated curve. In these gases, the analysis is complicated by the need to separate the bound electronic and rotational responses [27].

That the simple field-quadratic response of bound electrons continues to apply well beyond the ionization threshold is consistent with our Kramers-Kronig (KK) simulations presented in [9]. A physical interpretation of 
the KK results $[9,34]$ is that, in atoms dressed by the intense field, the change to the single-photon absorption coefficient (which contributes to the imaginary part of the effective susceptibility) is non-negligible and dominated by strong ac Stark shifts, which then causes the real nonlinear response (the real part of the effective susceptibility) to be quadratic in the field. This also applies to enhanced single-photon absorption from resonantly populated high lying states. For nonground state levels, the range of shifts can be as large as the ponderomotive energy, $U_{p} \sim 8 \mathrm{eV}$ at $120 \mathrm{TW} / \mathrm{cm}^{2}$, and population can be easily resonantly transferred to states within one photon from the continuum [35].

In summary, absolute measurements of ionization in $\mathrm{Ar}$, $\mathrm{Kr}, \mathrm{Xe}, \mathrm{N}_{2}$, and $\mathrm{O}_{2}$ have enabled absolute determination of the transient free and bound-electron contributions to the nonperturbative nonlinear polarizability. For our conditions, the bound component of the nonlinear polarizability is, to within our measurement accuracy, quadratic in the laser field amplitude over the full range of the interaction up to $>100 \mathrm{TW} / \mathrm{cm}^{2}$, which is well past the ionization threshold of the gases measured here and manifestly in the nonperturbative regime.

The authors thank I. Larkin, E. Rosenthal, N. Jhajj, and K. Y. Kim for discussions and technical assistance. J. K. W., S.Z., and H.M.M. acknowledge support by the Air Force Office of Scientific Research (FA9550-1610284, FA9550-16-10121), the Army Research Office (W911NF1410372), the Office of Naval Research (N00014-17-1-2705, N00014-17-1-2778), and the National Science Foundation (PHY1301948). A. B. and M.K. acknowledge support by the Air Force Office of Scientific Research (FA9550-16-10121).

*Corresponding author. milch@umd.edu

[1] C. G. Durfee, III., A. R. Rundquist, S. Backus, C. Herne, M. M. Murnane, and H. C. Kapteyn, Phys. Rev. Lett. 83, 2187 (1999).

[2] T. Popmintchev et al., Science 336, 1287 (2012).

[3] P. B. Corkum, C. Rolland, and T. Srinivasan-Rao, Phys. Rev. Lett. 57, 2268 (1986).

[4] A. Couairon, M. Franco, A. Mysyrowicz, J. Biegert, and U. Keller, Opt. Lett. 30, 2657 (2005).

[5] K. Y. Kim, I. Alexeev, and H. M. Milchberg, Appl. Phys. Lett. 81, 4124 (2002).

[6] A. Couairon and A. Mysyrowicz, Phys. Rep. 441, 47 (2007).

[7] L. Berge, S. Skupin, R. Nuter, J. Kasparian, and J.-P. Wolf, Rep. Prog. Phys. 70, 1633 (2007).

[8] J. K. Wahlstrand, Y.-H. Cheng, and H. M. Milchberg, Phys. Rev. A 85, 043820 (2012).

[9] J. K. Wahlstrand, Y.-H. Cheng, and H. M. Milchberg, Phys. Rev. Lett. 109, 113904 (2012).
[10] S. Zahedpour, J. K. Wahlstrand, and H. M. Milchberg, Opt. Lett. 40, 5794 (2015).

[11] A. Spott, A. Jaron-Becker, and A. Becker, Phys. Rev. A 90, 013426 (2014).

[12] B. Walker, B. Sheehy, L. F. DiMauro, P. Agostini, K. J. Schafer, and K. C. Kulander, Phys. Rev. Lett. 73, 1227 (1994).

[13] W. C. Wallace, O. Ghafur, C. Khurmi, S. Sainadh U, J. E. Calvert, D. E. Laban, M. G. Pullen, K. Bartschat, A. N. Grum-Grzhimailo, D. Wells, H. M. Quiney, X. M. Tong, I. V. Litvinyuk, R. T. Sang, and D. Kielpinski, Phys. Rev. Lett. 117, 053001 (2016).

[14] M. Richter, S. Patchkovskii, F. Morales, O. Smirnova, and M. Ivanov, New J. Phys. 15, 083012 (2013).

[15] P. Bejot, E. Cormier, E. Hertz, B. Lavorel, J. Kasparian, J.-P. Wolf, and O. Faucher, Phys. Rev. Lett. 110, 043902 (2013).

[16] V. Loriot, E. Hertz, O. Faucher, and B. Lavorel, Opt. Express 17, 13429 (2009).

[17] P. Bejot, J. Kasparian, S. Henin, V. Loriot, T. Vieillard, E. Hertz, O. Faucher, B. Lavorel, and J.-P. Wolf, Phys. Rev. Lett. 104, 103903 (2010).

[18] C. Bree, A. Demircan, and G. Steinmeyer, Phys. Rev. Lett. 106, 183902 (2011).

[19] M. Nurhuda, A. Suda, and K. Midorikawa, New J. Phys. 10, 053006 (2008).

[20] C. Kohler, R. Guichard, E. Lorin, S. Chelkowski, A. D. Bandrauk, L. Berge, and S. Skupin, Phys. Rev. A 87, 043811 (2013).

[21] J. K. Wahlstrand, S. Zahedpour, and H. M. Milchberg, J. Opt. Soc. Am. B 33, 1476 (2016).

[22] K. Y. Kim, I. Alexeev, and H. M. Milchberg, Opt. Express 10, 1563 (2002).

[23] Y.-H. Chen, S. Varma, I. Alexeev, and H. M. Milchberg, Opt. Express 15, 7458 (2007).

[24] Y.-H. Chen, S. Varma, A. York, and H. M. Milchberg, Opt. Express 15, 11341 (2007).

[25] J. K. Wahlstrand, Y.-H. Cheng, Y.-H. Chen, and H. M. Milchberg, Phys. Rev. Lett. 107, 103901 (2011).

[26] Y.-H. Cheng, N. Jhajj, J. K. Wahlstrand, and H. M. Milchberg, Opt. Express 21, 4740 (2013).

[27] See Supplemental Material at http://link.aps.org/ supplemental/10.1103/PhysRevLett.120.183901 for a discussion of experimental details, additional results for $\mathrm{Kr}$, $\mathrm{Xe}, \mathrm{N}_{2}$, and $\mathrm{O}_{2}$, and movies of $2 \mathrm{D}+1$ SSSI.

[28] A. M. Perelomov, V. S. Popov, and M. V. Terent'ev, Sov. Phys. JETP 23, 924 (1966).

[29] M. Kolesik and J. V. Moloney, Phys. Rev. E 70, 036604 (2004).

[30] J. Andreasen and M. Kolesik, Phys. Rev. E 86, 036706 (2012).

[31] A. Bahl, E. M. Wright, and M. Kolesik, Phys. Rev. A 94, 023850 (2016).

[32] M. Kolesik, J. M. Brown, A. Teleki, P. Jakobsen, J. V. Moloney, and E. M. Wright, Optica 1, 323 (2014).

[33] A. Bahl, J. K. Wahlstrand, S. Zahedpour, H. M. Milchberg, and M. Kolesik, Phys. Rev. A 96, 043867 (2017).

[34] M. Sheik-Bahae, D. C. Hutchings, D. J. Hagan, and E. W. Van Stryland, IEEE J. Quantum Electron. 27, 1296 (1991).

[35] M. P. de Boer and H. G. Muller, Phys. Rev. Lett. 68, 2747 (1992). 